\title{
A TWO-DIMENSIONAL RISK MODEL WITH PROPORTIONAL REINSURANCE
}

\author{
ANDREI L. BADESCU, ${ }^{*}$ University of Toronto \\ ERIC C. K. CHEUNG, ${ }^{* *}$ University of Hong Kong \\ LANDY RABEHASAINA, ${ }^{* * *}$ Université de Franche Comté
}

\begin{abstract}
In this paper we consider an extension of the two-dimensional risk model introduced in Avram, Palmowski and Pistorius (2008a). To this end, we assume that there are two insurers. The first insurer is subject to claims arising from two independent compound Poisson processes. The second insurer, which can be viewed as a different line of business of the same insurer or as a reinsurer, covers a proportion of the claims arising from one of these two compound Poisson processes. We derive the Laplace transform of the time until ruin of at least one insurer when the claim sizes follow a general distribution. The surplus level of the first insurer when the second insurer is ruined first is discussed at the end in connection with some open problems.
\end{abstract}

Keywords: Two-dimensional risk model; proportional reinsurance; geometric argument; absorbing set; time to ruin; deficit at ruin

2010 Mathematics Subject Classification: Primary 60G51

Secondary 60K30; 60J75

\section{Introduction}

Multidimensional risk theory has gained a lot of popularity in recent years. The problems that arise when studying the class of multivariate risk models involve an increased level of complexity when compared to the class of univariate risk models, mainly due to the dependence of claim severities and/or their interarrival times among the several lines of business under consideration. Historically, a quantity that has most frequently been treated in the univariate literature is the time to ruin, or the time of default, where ruin is defined as the first passage time below a certain threshold, with level 0 being the usual critical level. However, when considering multidimensional collective risk theory, ruin can be defined in several ways. The first time at least one of the risk processes falls below level 0, as well as the first time all the risk processes are below level 0 , are some particular examples for which bounds, asymptotic results, and extremely rare explicit solutions have been obtained for the ruin probabilities. Recent results pertaining to multidimensional risk models can be found in, e.g. Chan et al. (2003), Cai and Li (2005), (2007), Yuen et al. (2006), Li et al. (2007), Avram et al. (2008a), (2008b), Dang et al. (2009), Rabehasaina (2009), and Gong et al. (2010). Among these, the work that motivates the present work is that of Avram et al. (2008a). The authors considered the joint ruin

\footnotetext{
Received 23 September 2010; revision received 21 February 2011.

* Postal address: Department of Statistics, University of Toronto, 100 St. George Street, Toronto, Ontario, Canada. Email address: badescu@utstat.toronto.edu

** Postal address: Department of Statistics and Actuarial Science, University of Hong Kong, Pokfulam, Hong Kong. Email address: eckc@hku.hk

*** Postal address: Département de Mathématiques, Université de Franche Comté, 16 route de Gray, 25030 Besançon, France. Email address: 1rabehas@univ-fcomte.fr
} 
problem for two insurance companies that divide between them in different proportions both the premium income and the aggregate claims process which is modeled through a compound Poisson process. In practice, such a problem can be interpreted as an insurer-reinsurer scenario, where the reinsurer takes over a proportion of the insurer's losses. Restricting the claims to being exponentially distributed, Avram et al. (2008a) obtained an explicit analytic expression for the Laplace transform of the time to ruin, where ruin is defined as the first time at least one of the risk processes drops below 0. Using a geometric argument, the authors reduced the bivariate ruin problem to two distinct univariate problems, whose solutions are more easily obtainable. More specifically, Avram et al. (2008a) were able to find a (deterministic) critical time such that if ruin occurs after the critical time then it is caused by the first risk process, while if ruin occurs prior to the critical time then it is caused by the second risk process.

In this paper we generalize the work of Avram et al. (2008a) by considering a two-dimensional insurance risk model where one of the risk processes faces claims arising from two independent compound Poisson processes, of which only one is shared proportionally with the second risk process. As will be discussed in Section 2, such a risk model can be viewed in real life as a model for the surplus processes of two lines of business of the same company, or as an insurer-reinsurer application. Mathematically, the evolution of our two-dimensional risk process, denoted by $\left\{\left(Y_{t}^{1}, Y_{t}^{2}\right)\right\}_{t \geq 0}$, is described as

$$
\mathrm{d} Y_{t}^{1}=p_{1} \mathrm{~d} t-a \mathrm{~d} L_{t}-\mathrm{d} S_{t}, \quad \mathrm{~d} Y_{t}^{2}=p_{2} \mathrm{~d} t-(1-a) \mathrm{d} L_{t}, \quad\left(Y_{0}^{1}, Y_{0}^{2}\right)=\left(y_{1}, y_{2}\right),
$$

where $\left(y_{1}, y_{2}\right), y_{i} \geq 0(i=1,2)$, are the initial surpluses at time 0 and $\left(p_{1}, p_{2}\right), p_{i}>0$ $(i=1,2)$, are the premium rates. In addition, the aggregate claims processes $\left\{L_{t}\right\}_{t \geq 0}$ and $\left\{S_{t}\right\}_{t \geq 0}$ are given by

$$
L_{t}=\sum_{i=1}^{N_{t}^{L}} Z_{i}^{L} \quad \text { and } \quad S_{t}=\sum_{i=1}^{N_{t}^{S}} Z_{i}^{S}
$$

Here, $\left\{N_{t}^{L}\right\}_{t \geq 0}$ and $\left\{N_{t}^{S}\right\}_{t \geq 0}$ are Poisson processes with intensities $\lambda_{L}$ and $\lambda_{S}$, respectively, whereas $\left\{Z_{i}^{L}\right\}_{i=1}^{\infty}$ and $\left\{Z_{i}^{S}\right\}_{i=1}^{\infty}$ are the sequences of positive claims. It is assumed that $\left\{N_{t}^{L}\right\}_{t \geq 0}$, $\left\{N_{t}^{S}\right\}_{t \geq 0},\left\{Z_{i}^{L}\right\}_{i=1}^{\infty}$, and $\left\{Z_{i}^{S}\right\}_{i=1}^{\infty}$ are all mutually independent, and that $\left\{Z_{i}^{L}\right\}_{i=1}^{\infty}$ and $\left\{Z_{i}^{S}\right\}_{i=1}^{\infty}$ are independent and identically distributed sequences distributed as the generic random variables $Z^{L}$ and $Z^{S}$ with probability density functions (PDFs) $f_{L}(\cdot)$ and $f_{S}(\cdot)$, respectively. Note that we have relaxed Avram et al.'s (2008a) exponential claim size assumption to allow for arbitrary claim size distributions for both $Z^{L}$ and $Z^{S}$. From the dynamics of (1), it is clear that the first and second insurers share the compound Poisson process $\left\{L_{t}\right\}_{t \geq 0}$ at a constant ratio of $a$ and $1-a$, respectively, where $a \in(0,1)$, whereas $\left\{S_{t}\right\}_{t \geq 0}$ is covered entirely by the first insurer. The positive security loading conditions for each of the processes $\left\{Y_{t}^{1}\right\}_{t \geq 0}$ and $\left\{Y_{t}^{2}\right\}_{t \geq 0}$ are given by

$$
\begin{aligned}
& p_{1}>a \mathrm{E}\left[L_{1}\right]+\mathrm{E}\left[S_{1}\right], \\
& p_{2}>(1-a) \mathrm{E}\left[L_{1}\right],
\end{aligned}
$$

where $\mathrm{E}\left[L_{1}\right]$ and $\mathrm{E}\left[S_{1}\right]$ respectively represent the average aggregate claims per unit time arising from the processes $\left\{L_{t}\right\}_{t \geq 0}$ and $\left\{S_{t}\right\}_{t \geq 0}$. By defining the time to ruin for the $i$ th risk process as $\tau_{i}=\inf \left\{t \geq 0 \mid Y_{t}^{i}<0\right\}, i=1,2$, the key quantity of interest in this paper is the first time at which at least one of the surplus processes $\left\{Y_{t}^{1}\right\}_{t \geq 0}$ and $\left\{Y_{t}^{2}\right\}_{t \geq 0}$ becomes negative, namely,

$$
\tau=\inf \left\{t \geq 0 \mid \min \left(Y_{t}^{1}, Y_{t}^{2}\right)<0\right\}=\min \left(\tau_{1}, \tau_{2}\right)
$$


The paper is structured as follows. Using geometric arguments, in Section 2 we derive a sufficient set of constraints that will enable us to obtain an analytic solution for the Laplace transform of the time to ruin defined in (4). Section 3 gives two preliminary results required for the evaluation of the aforementioned Laplace transform, whose derivation is presented in Section 4. In Section 5 we briefly discuss the case where the ruin of $\left\{Y_{t}^{2}\right\}_{t \geq 0}$ occurs before the ruin of $\left\{Y_{t}^{1}\right\}_{t \geq 0}$ and present a few potential open problems.

\section{Model constraints via geometric interpretation}

In this section we employ a similar methodology to that in Avram et al. (2008a), i.e. we reduce the proposed two-dimensional risk model to more tractable univariate problems, for which various results in the literature can be exploited. To this end, in Figure 1 we present a sample path of the evolution of the two-dimensional risk model defined in (1). The vertical $y$-axis represents the surplus process $\left\{Y_{t}^{2}\right\}_{t \geq 0}$, whereas the horizontal $x$-axis represents $\left\{Y_{t}^{1}\right\}_{t \geq 0}$. We denote by $\Delta \subset \mathbb{R}^{2}$ the line whose equation is given by $y=(1-a) x / a$, and we let $\left\{X_{t}\right\}_{t \geq 0}$ be the process defined by $X_{t}=\left\langle\vec{v},\left(Y_{t}^{1}, Y_{t}^{2}\right)\right\rangle$ (with $\langle\cdot, \cdot\rangle$ denoting the usual scalar product), where $\vec{v}=(1-a,-a)$. Geometrically, $X_{t}$ can be seen as the algebraic distance (which is proportional to the Euclidean distance) between $\left(Y_{t}^{1}, Y_{t}^{2}\right) \in \mathbb{R}^{2}$ and $\Delta$, as illustrated in Figure 1. It satisfies

$$
\mathrm{d} X_{t}=\left[(1-a) p_{1}-a p_{2}\right] \mathrm{d} t-(1-a) \mathrm{d} S_{t}, \quad X_{0}=\left\langle\vec{v},\left(y_{1}, y_{2}\right)\right\rangle=(1-a) y_{1}-a y_{2} .
$$

The line $\Delta$ splits $\mathbb{R}^{2}$ into two disjoint sets, $\mathcal{A}^{+}$and $\mathcal{A}^{-}$, defined by

$$
\mathcal{A}^{+}:=\left\{\vec{x} \in \mathbb{R}^{2} \mid\langle\vec{x}, \vec{v}\rangle>0\right\} \quad \text { and } \quad \mathcal{A}^{-}:=\left\{\vec{x} \in \mathbb{R}^{2} \mid\langle\vec{x}, \vec{v}\rangle<0\right\} .
$$

The sets are such that $X_{t}>0$ is equivalent to $\left(Y_{t}^{1}, Y_{t}^{2}\right) \in \mathcal{A}^{+}$, and $X_{t}<0$ is equivalent to $\left(Y_{t}^{1}, Y_{t}^{2}\right) \in \mathcal{A}^{-}$. We further introduce the time of ruin of the newly defined univariate risk

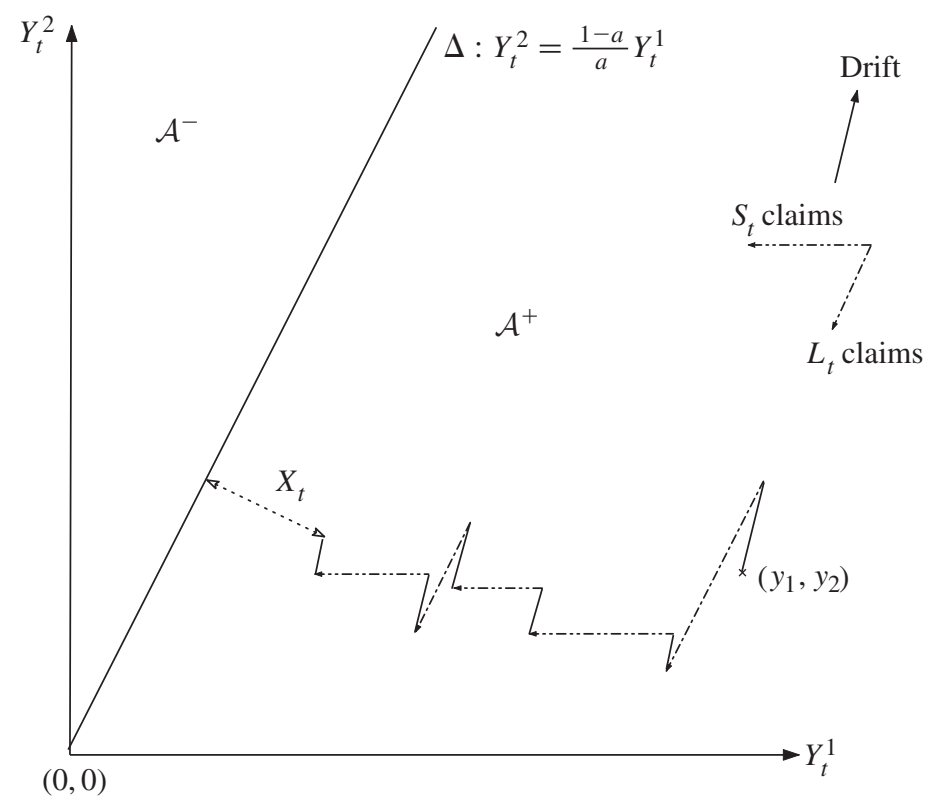

FIGURE 1: A sample path of $\left\{\left(Y_{t}^{1}, Y_{t}^{2}\right)\right\}_{t \geq 0}$. 
process $\left\{X_{t}\right\}_{t \geq 0}$ and denote it by $\tau_{X}=\inf \left\{t \geq 0 \mid X_{t}<0\right\}$. Without loss of generality and in order to avoid trivialities (as we will see later in the section), we let the two-dimensional risk process $\left\{\left(Y_{t}^{1}, Y_{t}^{2}\right)\right\}_{t \geq 0}$ start in the set $\mathcal{A}^{+}$, or, equivalently, we let $X_{0}=(1-a) y_{1}-a y_{2}>0$. The process $\left\{\left(Y_{t}^{1}, Y_{t}^{2}\right)\right\}_{t \geq 0}$ will then drift upwards as long as no claim occurs in any of the two individual risk processes $\left\{Y_{t}^{1}\right\}_{t \geq 0}$ and $\left\{Y_{t}^{2}\right\}_{t \geq 0}$. The occurrence of a claim in $\left\{L_{t}\right\}_{t \geq 0}$ will make $\left\{\left(Y_{t}^{1}, Y_{t}^{2}\right)\right\}_{t \geq 0}$ move downwards towards the origin $(0,0)$, parallel to the line $\Delta$, whereas the occurrence of a claim in $\left\{S_{t}\right\}_{t \geq 0}$ will make the process move to the left towards the vertical axis in parallel to the horizontal axis. The time of ruin defined in (4) can then be interpreted as the first time the two-dimensional risk process $\left\{\left(Y_{t}^{1}, Y_{t}^{2}\right)\right\}_{t \geq 0}$ exits the positive quadrant. Assuming that we start in $\mathcal{A}^{+}$, a closer look at Figure 1 reveals a competition between the first passage times $\tau_{X}$ and $\tau_{2}$ such that, if the process $\left\{\left(Y_{t}^{1}, Y_{t}^{2}\right)\right\}_{t \geq 0}$ downcrosses the horizontal axis before time $\tau_{X}$ (i.e. $\tau_{2}<\tau_{X}$ ) then ruin in the two-dimensional process will be caused purely by ruin in $\left\{Y_{t}^{2}\right\}_{t \geq 0}$ (i.e. $\tau=\tau_{2}$ ). A more challenging question arises in the opposite case when $\tau_{X} \leq \tau_{2}$. Under this scenario, in order to be able to analyze the time to ruin $\tau$ pertaining to the bivariate risk process defined in (1), we need to add an extra constraint that makes the set $\mathcal{A}^{-}$ an absorbing set, namely,

$$
\frac{p_{2}}{p_{1}}>\frac{1-a}{a},
$$

which will be assumed throughout the entire paper. Geometrically, condition (6) assumes that the slope of increase of the process $\left\{\left(Y_{t}^{1}, Y_{t}^{2}\right)\right\}_{t \geq 0}$ (during the collection of premium income) exceeds the slope of the line $\Delta$, ensuring that once $\left\{\left(Y_{t}^{1}, Y_{t}^{2}\right)\right\}_{t \geq 0}$ enters the set $\mathcal{A}^{-}$, it will never return to $\mathcal{A}^{+}$. Equivalently, condition (6) guarantees that $\left\{X_{t}\right\}_{t \geq 0}$ is a strictly decreasing process, which is evident from the dynamics (5). With (6) satisfied, the original two-dimensional ruin problem can be divided into two distinct univariate ruin problems as follows: $\tau_{2}<\tau_{X}$ implies that $\tau=\tau_{2}$, and $\tau_{2} \geq \tau_{X}$ implies that $\tau=\tau_{1}$. The present model generalizes that studied in Avram et al. (2008a), but the difficulty here arises from the fact that the deterministic critical time therein is now replaced by the stochastic time $\tau_{X}$. An important property that makes this approach tractable is that, under our construction, the processes $\left\{X_{t}\right\}_{t \geq 0}$ and $\left\{Y_{t}^{2}\right\}_{t \geq 0}$ are independent.

As previously mentioned in the introduction, we conclude this section with two practical insurance interpretations of the model defined in (1), together with further simplifications of the constraints introduced in (2), (3), and (6).

Interpretation $(i):\left\{Y_{t}^{1}\right\}_{t \geq 0}$ and $\left\{Y_{t}^{2}\right\}_{t \geq 0}$ are regarded as two lines of business of the same insurance company. That is, the two lines split $\left\{L_{t}\right\}_{t \geq 0}$ proportionally, while $\left\{S_{t}\right\}_{t \geq 0}$ is taken by line 1 only. The first line has possibly different loadings $\theta_{1}>0$ and $\theta_{2}>0$ on two different types of claim, so that

$$
p_{1}=\left(1+\theta_{1}\right) a \mathrm{E}\left[L_{1}\right]+\left(1+\theta_{2}\right) \mathrm{E}\left[S_{1}\right]
$$

while, for the second line, a different security loading $\theta_{3}>0$ is assumed such that

$$
p_{2}=\left(1+\theta_{3}\right)(1-a) \mathrm{E}\left[L_{1}\right] \text {. }
$$

Because the second line shares only a proportion of the $\left\{L_{t}\right\}_{t \geq 0}$ compound Poisson process, we assume that it has a larger loading, i.e. $\theta_{3}>\theta_{1}$. The total premium rate paid by policy holders for the aggregate claim process $\left\{L_{t}\right\}_{t \geq 0}$ is $\left[\left(1+\theta_{1}\right) a+\left(1+\theta_{3}\right)(1-a)\right] \mathrm{E}\left[L_{1}\right]$. It is clear that, under assumptions (7) and (8), the loading conditions (2) and (3) hold. Simple manipulations 
of (7) and (8) transform the constraint (6) into the condition

$$
\frac{\mathrm{E}\left[L_{1}\right]}{\mathrm{E}\left[S_{1}\right]}>\frac{1+\theta_{2}}{a\left(\theta_{3}-\theta_{1}\right)},
$$

which enables us to find the Laplace transform of the time to ruin $\tau$ (see Section 4). Note that, under condition (9), the process $\left\{L_{t}\right\}_{t \geq 0}$ may be generically interpreted as a riskier process than $\left\{S_{t}\right\}_{t \geq 0}$ in terms of the mean. Observe that in a practical situation the right-hand side of (9) will be larger than 1 .

Interpretation (ii): $\left\{Y_{t}^{1}\right\}_{t \geq 0}$ and $\left\{Y_{t}^{2}\right\}_{t \geq 0}$ represent the surplus processes of an insurer and a reinsurer, respectively. It is then assumed that the insurer receives premiums at loadings $\theta_{1}$ and $\theta_{2}$ for the aggregate claim processes $\left\{L_{t}\right\}_{t \geq 0}$ and $\left\{S_{t}\right\}_{t \geq 0}$, respectively. The insurer reinsures a proportion $1-a$ of the claims arising from $\left\{L_{t}\right\}_{t \geq 0}$, paying the reinsurer premiums at loading $\theta_{3}$. Then

$$
p_{1}=\left(1+\theta_{1}\right) \mathrm{E}\left[L_{1}\right]+\left(1+\theta_{2}\right) \mathrm{E}\left[S_{1}\right]-\left(1+\theta_{3}\right)(1-a) \mathrm{E}\left[L_{1}\right],
$$

whereas $p_{2}$ is the same as in (8). It is again assumed that $\theta_{3}>\theta_{1}$, otherwise the insurer may be tempted to reinsure the entire $\left\{L_{t}\right\}_{t \geq 0}$ while receiving arbitrage premium income. The premium rate paid by policy holders for $\left\{L_{t}\right\}_{t \geq 0}$ is just $\left(1+\theta_{1}\right) \mathrm{E}\left[L_{1}\right]$. Note that, under this possible practical interpretation, the positive security loading condition (2) does not necessarily hold, while (3) still holds. Further manipulations of (2) using (10) gives an equivalent condition

$$
\frac{\mathrm{E}\left[L_{1}\right]}{\mathrm{E}\left[S_{1}\right]}\left[\theta_{1}-\theta_{3}(1-a)\right]+\theta_{2}>0 .
$$

Using (8) and (10), the constraint condition (6) is transformed to

$$
\frac{\mathrm{E}\left[L_{1}\right]}{\mathrm{E}\left[S_{1}\right]}>\frac{1+\theta_{2}}{\theta_{3}-\theta_{1}} .
$$

As in the first practical interpretation, in practice $\left(1+\theta_{2}\right) /\left(\theta_{3}-\theta_{1}\right)>1$ in most of the cases, which means that condition (12) requires that the $\left\{L_{t}\right\}_{t \geq 0}$ process represents a larger risk, in terms of average, than the $\left\{S_{t}\right\}_{t \geq 0}$ process. Hence, it makes sense for the insurer to transfer part of the risk $\left\{L_{t}\right\}_{t \geq 0}$ to a reinsurer through reinsurance. Under the current insurer-reinsurer interpretation, condition (12) is compulsory to determine the Laplace transform of the time to ruin $\tau$ using geometric arguments, whereas relation (11) simply ensures that the insurer will not be ruined almost surely and that the problem makes sense from a practical point of view. Suppose that (11) holds. We need to distinguish between the following two cases.

1. If $1<\theta_{3} / \theta_{1} \leq 1 /(1-a)$ then condition (11) is automatically satisfied and, hence, only condition (12) is required.

2. If $\theta_{3} / \theta_{1}>1 /(1-a)$ then in order for both (11) and (12) to be satisfied, we require

$$
\frac{1+\theta_{2}}{\theta_{3}-\theta_{1}}<\frac{\mathrm{E}\left[L_{1}\right]}{\mathrm{E}\left[S_{1}\right]}<\frac{\theta_{2}}{\theta_{3}(1-a)-\theta_{1}} .
$$

If we relax the positive loading condition (11) (which is theoretically possible as all results obtained in the present paper apply even if (11) does not hold), the weaker condition $p_{1}>0$ will be required instead. Because of (10), such a weaker condition is translated to

$$
\frac{\mathrm{E}\left[L_{1}\right]}{\mathrm{E}\left[S_{1}\right]}\left[\theta_{3}-\theta_{1}-\left(1+\theta_{3}\right) a\right]<1+\theta_{2} \text {. }
$$


Again two cases need to be distinguished.

1. If $0<\theta_{3}-\theta_{1} \leq\left(1+\theta_{3}\right) a$ then (13) is satisfied automatically and, therefore, we need only (12).

2. If $\theta_{3}-\theta_{1}>\left(1+\theta_{3}\right) a$ then (12) together with (13) yields the condition

$$
\frac{1+\theta_{2}}{\theta_{3}-\theta_{1}}<\frac{\mathrm{E}\left[L_{1}\right]}{\mathrm{E}\left[S_{1}\right]}<\frac{1+\theta_{2}}{\theta_{3}-\theta_{1}-\left(1+\theta_{3}\right) a} .
$$

In the next section we analyze two important quantities for the independent processes $\left\{X_{t}\right\}_{t \geq 0}$ and $\left\{Y_{t}^{2}\right\}_{t \geq 0}$ that will help us to obtain the desired Laplace transform of the time to ruin $\tau$.

\section{Preliminary results}

As discussed in the previous section, the time to ruin $\tau_{X}$ in the process $\left\{X_{t}\right\}_{t \geq 0}$ plays a key role in the analysis. If ruin in the $\left\{X_{t}\right\}_{t \geq 0}$ process occurs prior to ruin in the $\left\{Y_{t}^{2}\right\}_{t \geq 0}$ process (i.e. $\tau_{X} \leq \tau_{2}$ ), then the knowledge of the surplus level $Y_{\tau_{X}}^{1}$ is of crucial importance because ruin of the bivariate process $\left\{\left(Y_{t}^{1}, Y_{t}^{2}\right)\right\}_{t \geq 0}$ will be due to the ruin of $\left\{Y_{t}^{1}\right\}_{t \geq 0}$ (i.e. $\tau=\tau_{1}$ ). It is easy to see that, under such a scenario, we have the relationship

$$
Y_{\tau_{X}}^{1}=\frac{a}{1-a} Y_{\tau_{X}}^{2}-\frac{1}{1-a}\left|X_{\tau_{X}}\right|,
$$

which expresses the surplus levels of the process $\left\{Y_{t}^{1}\right\}_{t \geq 0}$ in terms of those of $\left\{Y_{t}^{2}\right\}_{t \geq 0}$ and $\left\{X_{t}\right\}_{t \geq 0}$, all evaluated at time $\tau_{X}$. Driven by (14), in this section we derive two preliminary results.

First we are interested in the analysis of the time of ruin and the deficit at ruin in the $\left\{X_{t}\right\}_{t \geq 0}$ process. Under the current assumptions, $\left\{X_{t}\right\}_{t \geq 0}$ can be viewed as a risk process with positive initial surplus, negative drift (see the sufficient condition (6)), and downward jumps. Thus, ruin in the $\left\{X_{t}\right\}_{t \geq 0}$ process will occur almost surely, in two possible ways: due to jumps (i.e. $X_{\tau_{X}}<0$ ) or due to continuity (i.e. $X_{\tau_{X}}=0$ ). As a consequence, an important quantity that will be analyzed in Section 3.1 is the joint distribution of the time to ruin $\tau_{X}$ and the level of the deficit at ruin $\left|X_{\tau_{X}}\right|$.

When $\tau_{X} \leq \tau_{2}$, the level of the process $\left\{Y_{t}^{2}\right\}_{t \geq 0}$ at ruin time $\tau_{X}$ is also mandatory in the evaluation of (14). Thus, in Section 3.2 we present the distribution of the surplus process $\left\{Y_{t}^{2}\right\}_{t \geq 0}$ at a given time $t$, avoiding ruin enroute, namely $\mathrm{P}_{y_{2}}\left(\inf _{s \leq t} Y_{s}^{2}>0, Y_{t}^{2} \in \mathrm{d} u\right)$. The notation $\mathrm{P}_{y}(\cdot)$ represents the conditional probability given the initial surplus level $y$ at time 0 .

\subsection{The joint density of the time and deficit at ruin in $\left\{X_{t}\right\}_{t \geq 0}$}

Starting with (5), we rewrite its first equation as

$$
\mathrm{d} X_{t}=-c \mathrm{~d} t-\mathrm{d} S_{t}^{a}
$$

where $c=a p_{2}-(1-a) p_{1}>0$ and $S_{t}^{a}=(1-a) S_{t}$. Note that $\left\{S_{t}^{a}\right\}_{t \geq 0}$ is still a compound Poisson process with the same arrival rate $\lambda_{S}$ as $\left\{S_{t}\right\}_{t \geq 0}$, but with a scaled secondary generic random variable $Z^{a, S}=(1-a) Z^{S}$ having associated PDF $f_{a, S}(\cdot)$. Furthermore, we denote by $h_{C}(t \mid x)$ the density of $\tau_{X}$ at $t(0<t<x / c)$ for ruin by continuity given that $X_{0}=x$, and by $h_{J}(z, t \mid x)$ the joint density of $\left(\left|X_{\tau_{X}}\right|, \tau_{X}\right)$ at $(z, t)(0<t<x / c, z>0)$ for ruin by jumps given that $X_{0}=x$. The following proposition gives the above densities in explicit form, for generally distributed claim sizes. 
Proposition 1. In the risk process $\left\{X_{t}\right\}_{t \geq 0}$ defined by (15) with $X_{0}=x>0$, the joint distribution of the time of ruin $\tau_{X}$ and the deficit at ruin $\left|X_{\tau_{X}}\right|$ consists of

(i) contributions due to continuity (i.e. $\left|X_{\tau_{X}}\right|=0$ ):

(a) a point mass at $\tau_{X}=x / c$ with probability $\mathrm{P}_{x}\left(\tau_{X}=x / c,\left|X_{\tau_{X}}\right|=0\right)=\mathrm{e}^{-\lambda_{S} x / c}$,

(b) the density part of $\tau_{X}$ given by

$$
h_{C}(t \mid x)=c \sum_{n=1}^{\infty} \mathrm{e}^{-\lambda_{S} t} \frac{\left(\lambda_{S} t\right)^{n}}{n !} f_{a, S}^{* n}(x-c t)
$$

for $0<t<x / c$, where $f_{a, S}^{* n}(\cdot)$ is the $n$-fold convolution of $f_{a, S}(\cdot)$ with itself,

(ii) contributions due to jumps (i.e. $\left|X_{\tau_{X}}\right|>0$ ):

$$
\begin{aligned}
h_{J}(z, t \mid x)= & \lambda_{S} \mathrm{e}^{-\lambda_{S} t} f_{a, S}(z+x-c t) \\
& +\lambda_{S} \sum_{n=1}^{\infty} \mathrm{e}^{-\lambda_{S} t} \frac{\left(\lambda_{S} t\right)^{n}}{n !} \int_{0}^{x-c t} f_{a, S}(z+y) f_{a, S}^{* n}(x-c t-y) \mathrm{d} y
\end{aligned}
$$

for $0<t<x / c$ and $z>0$.

Proof. (i) In order to derive the joint distribution of the time to ruin and the deficit at ruin for $\left\{X_{t}\right\}_{t \geq 0}$, we first consider the Laplace transform of $\tau_{X}$ due to continuity, namely,

$$
\phi_{\beta, C}(x)=\mathrm{E}_{x}\left[\mathrm{e}^{-\beta \tau_{X}} \mathbf{1}_{\left\{X_{\tau_{X}}=0\right\}}\right],
$$

where $\beta \geq 0$ is the Laplace transform argument and $\mathrm{E}_{x}$ represents the conditional expectation given the initial surplus level $x$ at time 0 . By conditioning on the time and the size of the first claim, and using the Markov property of $\left\{X_{t}\right\}_{t \geq 0}$ followed by a change of variable, we obtain

$$
\begin{aligned}
\phi_{\beta, C}(x)= & \int_{x / c}^{\infty} \mathrm{e}^{-\beta(x / c)} \lambda_{S} \mathrm{e}^{-\lambda_{S} t} \mathrm{~d} t \\
& +\int_{0}^{x / c} \mathrm{e}^{-\beta t} \lambda_{S} \mathrm{e}^{-\lambda_{S} t} \int_{0}^{x-c t} \phi_{\beta, C}(x-c t-y) f_{a, S}(y) \mathrm{d} y \mathrm{~d} t \\
= & \mathrm{e}^{-\left(\lambda_{S}+\beta\right) x / c}+\frac{\lambda_{S}}{c} \int_{0}^{x} \mathrm{e}^{-\left(\lambda_{S}+\beta\right) v / c} \int_{0}^{x-v} \phi_{\beta, C}(x-v-y) f_{a, S}(y) \mathrm{d} y \mathrm{~d} v \\
= & \mathrm{e}^{-\left(\lambda_{S}+\beta\right) x / c}+\frac{\lambda_{S}}{c}\left(\mathrm{e}^{-\left(\lambda_{S}+\beta\right) \cdot / c} * \phi_{\beta, C} * f_{a, S}\right)(x),
\end{aligned}
$$

where ' $*$ ' is the convolution operator such that $\left(a_{1} * a_{2}\right)(y)=\int_{0}^{x} a_{1}(x-y) a_{2}(y) \mathrm{d} y(x \geq 0)$ for any functions $a_{1}(\cdot)$ and $a_{2}(\cdot)$ on $(0, \infty)$. If we define the Laplace transforms

$$
\tilde{\phi}_{\beta, C}(s)=\int_{0}^{\infty} \mathrm{e}^{-s x} \phi_{\beta, C}(x) \mathrm{d} x \quad \text { and } \quad \tilde{f}_{a, S}(s)=\int_{0}^{\infty} \mathrm{e}^{-s x} f_{a, S}(x) \mathrm{d} x,
$$

taking an extra Laplace transform with respect to $x$ in the above equation and then solving for $\tilde{\phi}_{\beta, C}(s)$ leads to

$$
\begin{aligned}
\tilde{\phi}_{\beta, C}(s) & =\frac{1}{\left(\lambda_{S}+\beta\right) / c+s} /\left(1-\frac{\lambda_{S}}{c} \frac{1}{\left(\lambda_{S}+\beta\right) / c+s} \tilde{f}_{a, S}(s)\right) \\
& =\frac{1}{\left(\lambda_{S}+\beta\right) / c+s}+\sum_{n=1}^{\infty}\left(\frac{\lambda_{S}}{c}\right)^{n} \frac{1}{\left[\left(\lambda_{S}+\beta\right) / c+s\right]^{n+1}}\left[\tilde{f}_{a, S}(s)\right]^{n} .
\end{aligned}
$$


Therefore, via Laplace transform inversion with respect to $s$, we arrive at

$$
\begin{aligned}
\phi_{\beta, C}(x) & =\mathrm{e}^{-\left(\lambda_{S}+\beta\right) x / c}+\sum_{n=1}^{\infty}\left(\frac{\lambda_{S}}{c}\right)^{n} \int_{0}^{x} \frac{y^{n} \mathrm{e}^{-\left(\lambda_{S}+\beta\right) y / c}}{n !} f_{a, S}^{* n}(x-y) \mathrm{d} y \\
& =\mathrm{e}^{-\left(\lambda_{S}+\beta\right) x / c}+c \sum_{n=1}^{\infty} \frac{\lambda_{S}^{n}}{n !} \int_{0}^{x / c} \mathrm{e}^{-\left(\lambda_{S}+\beta\right) t} t^{n} f_{a, S}^{* n}(x-c t) \mathrm{d} t .
\end{aligned}
$$

An extra Laplace transform inversion with respect to $\beta$ yields the desired result in (i). Probabilistically, parts (a) and (b) can be interpreted as follows.

(a) The process $\left\{X_{t}\right\}_{t \geq 0}$ decreases continuously for a length of time $x / c$ without any claim in the interim.

(b) The density part (16) arises when there is at least one jump (i.e. $n \geq 1$ ) before ruin by continuity. The term $\mathrm{e}^{-\lambda_{S} t}\left(\lambda_{S} t\right)^{n} / n$ ! is the probability that there are $n$ jumps until time $t$, and the sum of the $n$ jumps should be exactly $x-c t$ so that ruin occurs by continuity at time $t$, giving rise to the term $f_{a, S}^{* n}(x-c t)$. The factor $c$ reflects a change in unit.

(ii) Similarly, we consider the quantity for the ruin of $\left\{X_{t}\right\}_{t \geq 0}$ due to jumps, defined by

$$
\phi_{\beta, J}(x)=\mathrm{E}_{x}\left[\mathrm{e}^{-\beta \tau_{X}} w\left(\left|X_{\tau_{X}}\right|\right) \mathbf{1}_{\left\{X_{\tau_{X}}<0\right\}}\right],
$$

where $w(\cdot)$ is a penalty function that depends only on the deficit at ruin $\left|X_{\tau_{X}}\right|$. Conditioning again on the time and the size of the first claim, we obtain

$$
\begin{aligned}
\phi_{\beta, J}(x)= & \int_{0}^{x / c} \mathrm{e}^{-\beta t} \lambda_{S} \mathrm{e}^{-\lambda_{S} t} \int_{0}^{x-c t} \phi_{\beta, J}(x-c t-y) f_{a, S}(y) \mathrm{d} y \mathrm{~d} t \\
& +\int_{0}^{x / c} \mathrm{e}^{-\beta t} \lambda_{S} \mathrm{e}^{-\lambda_{S} t} \int_{x-c t}^{\infty} w(y-(x-c t)) f_{a, S}(y) \mathrm{d} y \mathrm{~d} t \\
= & \frac{\lambda_{S}}{c}\left(\mathrm{e}^{-\left(\lambda_{S}+\beta\right) \cdot / c} * \phi_{\beta, J} * f_{a, S}\right)(x)+\frac{\lambda_{S}}{c}\left(\mathrm{e}^{-\left(\lambda_{S}+\beta\right) \cdot c} * \omega_{a}\right)(x),
\end{aligned}
$$

where

$$
\omega_{a}(x)=\int_{x}^{\infty} w(y-x) f_{a, S}(y) \mathrm{d} y .
$$

Taking further Laplace transforms yields

$\tilde{\phi}_{\beta, J}(s)=\frac{\lambda_{S}}{c} \frac{1}{\left(\lambda_{S}+\beta\right) / c+s} \tilde{\omega}_{a}(s) /\left(1-\frac{\lambda_{S}}{c} \frac{1}{\left(\lambda_{S}+\beta\right) / c+s} \tilde{f}_{a, S}(s)\right)=\frac{\lambda_{S}}{c} \tilde{\omega}_{a}(s) \tilde{\phi}_{\beta, C}(s)$,

where $\tilde{\phi}_{\beta, J}(s)=\int_{0}^{\infty} \mathrm{e}^{-s x} \phi_{\beta, J}(x) \mathrm{d} x$ and $\tilde{\omega}_{a}(s)=\int_{0}^{\infty} \mathrm{e}^{-s x} \omega_{a}(x) \mathrm{d} x$. Hence, inversion with respect to $\beta$ leads to

$$
\begin{aligned}
\phi_{\beta, J}(x) & =\frac{\lambda_{S}}{c} \int_{0}^{x} \omega_{a}(y) \phi_{\beta, C}(x-y) \mathrm{d} y \\
& =\int_{0}^{\infty} w(z)\left(\frac{\lambda_{S}}{c} \int_{0}^{x} f_{a, S}(z+y) \phi_{\beta, C}(x-y) \mathrm{d} y\right) \mathrm{d} z
\end{aligned}
$$


where the second equality follows from substitution of (19). Using (18) followed by some manipulations, we find that

$$
\begin{aligned}
\frac{\lambda_{S}}{c} \int_{0}^{x} & f_{a, S}(z+y) \phi_{\beta, C}(x-y) \mathrm{d} y \\
= & \lambda_{S} \int_{0}^{x / c} \mathrm{e}^{-\left(\lambda_{S}+\beta\right) t} f_{a, S}(z+x-c t) \mathrm{d} t \\
& \quad+\lambda_{S} \sum_{n=1}^{\infty} \frac{\lambda_{S}^{n}}{n !} \int_{0}^{x / c} \mathrm{e}^{-\left(\lambda_{S}+\beta\right) t} t^{n} \int_{0}^{x-c t} f_{a, S}(z+y) f_{a, S}^{* n}(x-c t-y) \mathrm{d} y \mathrm{~d} t .
\end{aligned}
$$

Therefore, we finally observe that $\phi_{\beta, J}(x)$ admits the representation

$$
\phi_{\beta, J}(x)=\int_{0}^{\infty} \int_{0}^{x / c} \mathrm{e}^{-\beta t} w(z) h_{J}(z, t \mid x) \mathrm{d} t \mathrm{~d} z,
$$

where $h_{J}(z, t \mid x)$ is the joint density of $\left(\left|X_{\tau_{X}}\right|, \tau_{X}\right)$ at $(z, t)$ given in part (ii). Probabilistically, the density (17) can be interpreted as follows. The first term $\lambda_{S} \mathrm{e}^{-\lambda_{S} t} f_{a, S}(z+x-c t)$ is the case where only one jump causes ruin. Such a jump occurs at time $t$, and the size of the jump should be $z+x-c t$ in order to result in a deficit of $z$. The second term reflects the cases where $n+1$ jumps cause ruin $(n \geq 1)$. In this case, there are $n$ jumps by time $t$ with probability $\mathrm{e}^{-\lambda_{S} t}\left(\lambda_{S} t\right)^{n} / n$ !, and the sum of these $n$ jumps should be $x-c t-y$ for some $0<y<x-c t$, contributing to the term $f_{a, S}^{* n}(x-c t-y)$. At this moment the process is at level $y$, then the $(n+1)$ th jump occurs at rate $\lambda_{S}$ and it should be of size $z+y$ to cause a deficit of $z$.

Remark 1. The convolution terms and, hence, the integrals in (16) and (17) can be explicitly evaluated when the density $f_{a, S}(\cdot)$ belongs to, for example, the class of mixed Erlang distributions (see Dickson and Willmot (2005)). Such a class is not only dense in the set of positive continuous distributions (see Tijms (1994, pp. 163-164)), but also contains many other distributions, some of which are nontrivial, as special cases (see Willmot and Woo (2007)).

\subsection{The density of $\left\{Y_{t}^{2}\right\}_{t \geq 0}$, avoiding ruin enroute}

Similarly to the way in which we rewrote the process $\left\{X_{t}\right\}_{t \geq 0}$ in (15), we write

$$
\mathrm{d} Y_{t}^{2}=p_{2} \mathrm{~d} t-\mathrm{d} L_{t}^{a}
$$

where $L_{t}^{a}=(1-a) L_{t}$. Here $\left\{L_{t}^{a}\right\}_{t \geq 0}$ is a compound Poisson process with the same arrival rate $\lambda_{L}$ as in the original $\left\{L_{t}\right\}_{t \geq 0}$ process, but with a scaled secondary generic random variable $Z^{a, L}=(1-a) Z^{L}$ having associated PDF $f_{a, L}(\cdot)$. The following proposition recovers the density of the process $\left\{Y_{t}^{2}\right\}_{t \geq 0}$, avoiding ruin enroute.

Proposition 2. In the risk process $\left\{Y_{t}^{2}\right\}_{t \geq 0}$ defined by (20), the distribution of the surplus process $\left\{Y_{t}^{2}\right\}_{t \geq 0}$ at level $u$, at a given time $t$, avoiding ruin enroute consists of the following contributions.

(i) A point mass given by

$$
\mathrm{P}_{y_{2}}\left(\inf _{s \leq t} Y_{s}^{2}>0, Y_{t}^{2}=u\right)=\mathrm{e}^{-\lambda_{L} t}
$$

for $u=y_{2}+p_{2} t$. 
(ii) The density part given by

$$
\mathrm{P}_{y_{2}}\left(\inf _{s \leq t} Y_{s}^{2}>0, Y_{t}^{2} \in \mathrm{d} u\right)=\zeta\left(y_{2}, t, u\right) \mathrm{d} u
$$

for $u<y_{2}+p_{2} t$, where

$$
\zeta\left(y_{2}, t, u\right) \quad\left\{\begin{array}{c}
\mathrm{e}^{-\lambda_{L} t}\left(b\left(y_{2}, p_{2} t, u\right)\right. \\
+\sum_{n=1}^{\infty} \frac{\left(\lambda_{L} t\right)^{n}}{n !} \\
\left.\quad \times \int_{0}^{p_{2} t} \frac{z}{p_{2} t} f_{a, L}^{* n}\left(p_{2} t-z\right) b\left(y_{2}, z, u\right) \mathrm{d} z\right), \quad u \leq y_{2} ; t>0, \\
\mathrm{e}^{-\lambda_{L} t}\left[\begin{array}{r}
b\left(y_{2}, p_{2} t, u\right) \\
+\sum_{n=1}^{\infty} \frac{\left(\lambda_{L} t\right)^{n}}{n !}\left(\frac{u-y_{2}}{p_{2} t} f_{a, L}^{* n}\left(p_{2} t-\left(u-y_{2}\right)\right)\right. \\
\left.\left.\quad+\int_{u-y_{2}}^{p_{2} t} \frac{z}{p_{2} t} f_{a, L}^{* n}\left(p_{2} t-z\right) b\left(y_{2}, z, u\right) \mathrm{d} z\right)\right], \quad u>y_{2} ; t>\frac{u-y_{2}}{p_{2}},
\end{array}\right.
\end{array}\right.
$$

$b\left(y_{2}, z, u\right)$

$$
= \begin{cases}\sum_{n=1}^{\infty}\left(\frac{\lambda_{L}}{p_{2}}\right)^{n} \int_{0}^{z \wedge u} \xi_{n}\left(y_{2}-u+v, z-v\right) \mathrm{d} v, & u \leq y_{2} ; z>0, \\ \sum_{n=1}^{\infty}\left(\frac{\lambda_{L}}{p_{2}}\right)^{n} \int_{0}^{y_{2}+((z-u) \wedge 0)} \xi_{n}\left(v, y_{2}+z-u-v\right) \mathrm{d} v, & u>y_{2} ; z>u-y_{2},\end{cases}
$$

and

$$
\begin{aligned}
\xi_{n}\left(y_{2}, z\right)= & \frac{y_{2}^{n-1}}{(n-1) !} f_{a, L}^{* n}\left(z+y_{2}\right) \\
& +\sum_{j=1}^{n-1}\left(\begin{array}{l}
n \\
j
\end{array}\right) \frac{(-1)^{j}}{(n-1) !} \int_{0}^{y_{2}} v^{n-1} f_{a, L}^{* j}\left(y_{2}-v\right) f_{a, L}^{*(n-j)}(z+v) \mathrm{d} v .
\end{aligned}
$$

Proof. Our goal here is to perform Laplace transform inversion on the expression

$$
\int_{t=0}^{\infty} \mathrm{e}^{-\beta t} \mathrm{P}_{y_{2}}\left(\inf _{s \leq t} Y_{s}^{2}>0, Y_{t}^{2} \in \mathrm{d} u\right) \mathrm{d} t=\left[\mathrm{e}^{-\rho u} W^{(\beta)}\left(y_{2}\right)-\mathbf{1}_{\left\{y_{2} \geq u\right\}} W^{(\beta)}\left(y_{2}-u\right)\right] \mathrm{d} u .
$$

See Suprun (1976) and Bertoin (1997, Lemma 1). Here $W^{(\beta)}(\cdot)$ is the $\beta$-scale function of $\left\{Y_{t}^{2}\right\}_{t \geq 0}$ with Laplace transform

$$
\int_{0}^{\infty} \mathrm{e}^{-s x} W^{(\beta)}(x) \mathrm{d} x=\frac{1}{p_{2} s-\left(\lambda_{L}+\beta\right)+\lambda_{L} \tilde{f}_{a, L}(s)}, \quad s>\rho,
$$


where $\tilde{f}_{a, L}(s)=\int_{0}^{\infty} \mathrm{e}^{-s x} f_{a, L}(x) \mathrm{d} x$, and the quantity $\rho$ appearing in (26) is the unique nonnegative root to the equation (in $\xi$ )

$$
p_{2} \xi-\left(\lambda_{L}+\beta\right)+\lambda_{L} \tilde{f}_{a, L}(\xi)=0 .
$$

Next, we denote by $g_{\beta}\left(u \mid y_{2}\right)$ the so-called discounted density of the surplus prior to ruin $Y_{\tau_{2}-}^{2}$ at $u$ for the process $\left\{Y_{t}^{2}\right\}_{t \geq 0}$, given an initial capital of $Y_{0}^{2}=y_{2}$ :

$$
g_{\beta}\left(u \mid y_{2}\right) \mathrm{d} u=\int_{t=0}^{\infty} \mathrm{e}^{-\beta t} \mathrm{P}_{y_{2}}\left(Y_{\tau_{2}-}^{2} \in \mathrm{d} u, \tau_{2} \in \mathrm{d} t\right)
$$

By integrating out the second argument of Equation (41) of Cheung and Landriault (2010), we have

$$
g_{\beta}\left(u \mid y_{2}\right)=\frac{\lambda_{L}}{p_{2}}\left[\mathrm{e}^{-\rho u} v_{\beta}\left(y_{2}\right)-\mathbf{1}_{\left\{y_{2} \geq u\right\}} v_{\beta}\left(y_{2}-u\right)\right] \bar{F}_{a, L}(u)
$$

where the function $v_{\beta}(\cdot)$ is related to $W^{(\beta)}(\cdot)$ via $v_{\beta}(\cdot)=p_{2} W^{(\beta)}(\cdot)$ (which is evident by comparing Equation (11) of Cheung and Landriault (2010) with (27)), and $\bar{F}_{a, L}(\cdot)$ is the survival function of the generic random variable $Z^{a, L}$. Thus, using (26), we can express the above equation as

$$
g_{\beta}\left(u \mid y_{2}\right) \mathrm{d} u=\lambda_{L}\left[\int_{t=0}^{\infty} \mathrm{e}^{-\beta t} \mathrm{P}_{y_{2}}\left(\inf _{s \leq t} Y_{s}^{2}>0, Y_{t}^{2} \in \mathrm{d} u\right) \mathrm{d} t\right] \bar{F}_{a, L}(u) .
$$

On the other hand, Corollary 1 of Landriault and Willmot (2009) implies that

$g_{\beta}\left(u \mid y_{2}\right)=\lambda_{L}\left[\mathrm{e}^{-\beta\left(u-y_{2}\right) / p_{2}}\left(\frac{1}{p_{2}} \mathrm{e}^{-\lambda_{L}\left(u-y_{2}\right) / p_{2}} \mathbf{1}_{\left\{u>y_{2}\right\}}\right)+\int_{0}^{\infty} \mathrm{e}^{-\beta t} \zeta\left(y_{2}, t, u\right) \mathrm{d} t\right] \bar{F}_{a, L}(u)$,

where $\zeta\left(y_{2}, t, u\right)$ is defined through (23), (24), and (25). Hence, by the uniqueness of Laplace transforms, a comparison between (29) and (30) yields the desired result in (21) and (22).

Remark 2. Instead of drawing connections between the existing results (26) and (28) to prove (29), we also provide a probabilistic proof as follows. In order to ensure that the surplus prior to ruin $Y_{\tau_{2}-}^{2}$ is $u$, the process $\left\{Y_{t}^{2}\right\}_{t \geq 0}$ has to first reach level $u$ from level $y_{2}$ at some time $t$ without ruin occurring in the interim, which is represented by the term $\mathrm{P}_{y_{2}}\left(\inf _{s \leq t} Y_{s}^{2}>0, Y_{t}^{2} \in \mathrm{d} u\right.$ ). Being at level $u$, if a claim occurs at the next instant (with probability $\lambda_{L} \mathrm{~d} t$ ) and such a claim is larger then $u$ (with probability $\bar{F}_{a, L}(u)$ ), then the time of ruin of $\left\{Y_{t}^{2}\right\}_{t \geq 0}$ is $t$. Since we are concerned with discounted density here, we need to multiply by $\mathrm{e}^{-\beta t}$ and integrate with respect to $t$, resulting in (29).

Remark 3. The quantity $\zeta\left(y_{2}, t, u\right)$ can be explicitly evaluated when $f_{a, L}(\cdot)$ follows a mixed Erlang distribution using the results in Landriault and Willmot (2009, Section 4).

\section{The Laplace transform of the joint ruin time}

In this section we give the main result of the paper regarding the Laplace transform of the time to ruin $\tau$, namely $\mathrm{E}_{\left(y_{1}, y_{2}\right)}\left[\mathrm{e}^{-\beta \tau} \mathbf{1}_{\{\tau<\infty\}}\right]$, where $\mathrm{E}_{\left(y_{1}, y_{2}\right)}$ is the conditional expectation given the initial surplus levels $\left(Y_{0}^{1}, Y_{0}^{2}\right)=\left(y_{1}, y_{2}\right)$. 
Proposition 3. In the bivariate risk process $\left\{\left(Y_{t}^{1}, Y_{t}^{2}\right)\right\}_{t \geq 0}$ defined by (1) with $x=(1-a) y_{1}-$ $a y_{2}>0$, the Laplace transform of the time to ruin is given by

$$
\mathrm{E}_{\left(y_{1}, y_{2}\right)}\left[\mathrm{e}^{-\beta \tau} \mathbf{1}_{\{\tau<\infty\}}\right]=\mathrm{E}_{\left(y_{1}, y_{2}\right)}\left[\mathrm{e}^{-\beta \tau_{2}} \mathbf{1}_{\left\{\tau_{X}>\tau_{2}\right\}}\right]+\mathrm{E}_{\left(y_{1}, y_{2}\right)}\left[\mathrm{e}^{-\beta \tau_{1}} \mathbf{1}_{\left\{\tau_{X} \leq \tau_{2}, \tau_{1}<\infty\right\}}\right] .
$$

The first part in (31) can be evaluated as

$$
\mathrm{E}_{\left(y_{1}, y_{2}\right)}\left[\mathrm{e}^{-\beta \tau_{2}} \mathbf{1}_{\left\{\tau_{X}>\tau_{2}\right\}}\right]=\int_{0}^{x / c} \mathrm{e}^{-\beta t} \mathrm{P}_{x}\left(\tau_{X}>t\right) \mathrm{P}_{y_{2}}\left(\tau_{2} \in \mathrm{d} t\right),
$$

where, for $0<t<x / c$,

$$
\mathrm{P}_{x}\left(\tau_{X}>t\right)=\sum_{n=0}^{\infty} \mathrm{e}^{-\lambda_{S} t} \frac{\left(\lambda_{S} t\right)^{n}}{n !} F_{a, S}^{* n}(x-c t)
$$

whereas $\mathrm{P}_{y_{2}}\left(\tau_{2} \in \mathrm{d} t\right)$ is simply the density of the time of ruin $\tau_{2}$ of $\left\{Y_{t}^{2}\right\}_{t \geq 0}$ and is given in Dickson and Willmot (2005). Here $F_{a, S}^{* n}(\cdot)$ is the cumulative distribution function corresponding to the PDF $f_{a, S}^{* n}(\cdot)$ with the usual convention that $F_{a, S}^{* 0}(v)=\mathbf{1}_{\{v \geq 0\}}$.

Next, we denote by $\mathrm{E}_{y_{1}}\left[\mathrm{e}^{-\beta \tau_{1}} \mathbf{1}_{\left\{\tau_{1}<\infty\right\}}\right]$ the Laplace transform of the time of ruin $\tau_{1}$ for $\left\{Y_{t}^{1}\right\}_{t \geq 0}$, which is known to be the tail of a compound geometric distribution under $\beta>0$, or, for any $\beta \geq 0$, if the positive loading condition (2) holds (see Lin and Willmot (1999, Section 2)). Then the second part of (31), namely $\mathrm{E}_{\left(y_{1}, y_{2}\right)}\left[\mathrm{e}^{-\beta \tau_{1}} \mathbf{1}_{\left\{\tau_{X} \leq \tau_{2}, \tau_{1}<\infty\right\}}\right]$, is the sum of the six contributions given below.

(i) For ruin of $\left\{X_{t}\right\}_{t \geq 0}$ due to continuity, we have the following contributions.

(a) No claims from $\left\{S_{t}\right\}_{t \geq 0}$ and no claims from $\left\{L_{t}\right\}_{t \geq 0}$ in $\left(0, \tau_{X}\right]$ :

$$
\mathrm{e}^{-\left(\beta+\lambda_{S}+\lambda_{L}\right) x / c} \mathrm{E}_{a\left(y_{2}+p_{2} x / c\right) /(1-a)}\left[\mathrm{e}^{-\beta \tau_{1}} \mathbf{1}_{\left\{\tau_{1}<\infty\right\}}\right] .
$$

(b) No claims from $\left\{S_{t}\right\}_{t \geq 0}$ and at least one claim from $\left\{L_{t}\right\}_{t \geq 0}$ in $\left(0, \tau_{X}\right]$ :

$$
\int_{0}^{y_{2}+p_{2} x / c} \mathrm{e}^{-\left(\beta+\lambda_{S}\right) x / c} \mathrm{E}_{a u /(1-a)}\left[\mathrm{e}^{-\beta \tau_{1}} \mathbf{1}_{\left\{\tau_{1}<\infty\right\}}\right] \zeta\left(y_{2}, \frac{x}{c}, u\right) \mathrm{d} u .
$$

(c) At least one claim from $\left\{S_{t}\right\}_{t \geq 0}$ and no claims from $\left\{L_{t}\right\}_{t \geq 0}$ in $\left(0, \tau_{X}\right]$ :

$$
\int_{0}^{x / c} \mathrm{e}^{-\left(\beta+\lambda_{L}\right) t} \mathrm{E}_{a\left(y_{2}+p_{2} t\right) /(1-a)}\left[\mathrm{e}^{-\beta \tau_{1}} \mathbf{1}_{\left\{\tau_{1}<\infty\right\}}\right] h_{C}(t \mid x) \mathrm{d} t .
$$

(d) At least one claim from $\left\{S_{t}\right\}_{t \geq 0}$ and at least one claim from $\left\{L_{t}\right\}_{t \geq 0}$ in $\left(0, \tau_{X}\right]$ :

$$
\int_{0}^{x / c} \mathrm{e}^{-\beta t}\left(\int_{0}^{y_{2}+p_{2} t} \mathrm{E}_{a u /(1-a)}\left[\mathrm{e}^{-\beta \tau_{1}} \mathbf{1}_{\left\{\tau_{1}<\infty\right\}}\right] \zeta\left(y_{2}, t, u\right) \mathrm{d} u\right) h_{C}(t \mid x) \mathrm{d} t .
$$

(ii) For ruin of $\left\{X_{t}\right\}_{t \geq 0}$ due to jumps, we have the following contributions.

(a) No claims from $\left\{L_{t}\right\}_{t \geq 0}$ in $\left(0, \tau_{X}\right]$ :

$$
\begin{aligned}
\int_{0}^{x / c} \mathrm{e}^{-\left(\beta+\lambda_{L}\right) t}\left(\int_{0}^{a\left(y_{2}+p_{2} t\right)}\right. & \mathrm{E}_{a\left(y_{2}+p_{2} t\right) /(1-a)-z /(1-a)}\left[\mathrm{e}^{-\beta \tau_{1}} \mathbf{1}_{\left\{\tau_{1}<\infty\right\}}\right] \\
& \left.\times h_{J}(z, t \mid x) \mathrm{d} z+\int_{a\left(y_{2}+p_{2} t\right)}^{\infty} h_{J}(z, t \mid x) \mathrm{d} z\right) \mathrm{d} t .
\end{aligned}
$$


(b) At least one claim from $\left\{L_{t}\right\}_{t \geq 0}$ in $\left(0, \tau_{X}\right]$ :

$$
\begin{gathered}
\int_{0}^{x / c} \mathrm{e}^{-\beta t} \int_{0}^{y_{2}+p_{2} t}\left(\int_{0}^{a u} \mathrm{E}_{a u /(1-a)-z /(1-a)}\left[\mathrm{e}^{-\beta \tau_{1}} \mathbf{1}_{\left\{\tau_{1}<\infty\right\}}\right] h_{J}(z, t \mid x) \mathrm{d} z\right. \\
\left.+\int_{a u}^{\infty} h_{J}(z, t \mid x) \mathrm{d} z\right) \zeta\left(y_{2}, t, u\right) \mathrm{d} u \mathrm{~d} t
\end{gathered}
$$

Proof. The decomposition in (31) is a direct consequence of the fact that $\tau_{X}>\tau_{2}$ implies that $\tau=\tau_{2}$, whereas $\tau_{X} \leq \tau_{2}$ implies that $\tau=\tau_{1}$. Equation (32) follows by conditioning on $\tau_{2}$ along with the independence of the processes $\left\{X_{t}\right\}_{t \geq 0}$ and $\left\{Y_{t}^{2}\right\}_{t \geq 0}$. Equation (33) can be argued probabilistically as follows. The term $\mathrm{e}^{-\lambda_{S} t}\left(\lambda_{S} t\right)^{n} / n$ ! represents the probability that there are $n$ jumps until time $t$. In order for $\left\{Y_{t}^{2}\right\}_{t \geq 0}$ to survive at time $t(0<t<x / c)$, the sum of the $n$ jumps should be no larger than $x-c t$, resulting in the term $F_{a, S}^{* n}(x-c t)$. Summing over all possible $n$ (i.e. $n \geq 0$ ) yields the desired result.

For the second term $\mathrm{E}_{\left(y_{1}, y_{2}\right)}\left[\mathrm{e}^{-\beta \tau_{1}} \mathbf{1}_{\left\{\tau_{X} \leq \tau_{2}, \tau_{1}<\infty\right\}}\right]$ in (31), we note that, when $\tau_{X} \leq \tau_{2}$, at time $\tau_{X}$ the process $\left\{Y_{t}^{1}\right\}_{t \geq 0}$ is either on the borderline $\Delta$ of the absorbing set $\mathcal{A}^{-}$(i.e. $\left\{X_{t}\right\}_{t \geq 0}$ ruins due to continuity) or inside the set $\mathcal{A}^{-}$(i.e. $\left\{X_{t}\right\}_{t \geq 0}$ ruins due to jumps). Moreover, to keep track of the ruin times $\tau_{X}$ and $\tau_{2}$ we also recall that $\left\{X_{t}\right\}_{t \geq 0}$ is only subject to claims from $\left\{S_{t}\right\}_{t \geq 0}$ whereas $\left\{Y_{t}^{2}\right\}_{t \geq 0}$ is only subject to claims from $\left\{L_{t}\right\}_{t \geq 0}$. For ruin of $\left\{X_{t}\right\}_{t \geq 0}$ due to continuity, we have $Y_{\tau_{X}}^{1}=a Y_{\tau_{X}}^{2} /(1-a)$. This case is analyzed based on the number of claims arising from $\left\{S_{t}\right\}_{t \geq 0}$ and $\left\{L_{t}\right\}_{t \geq 0}$, respectively, in the interval $\left(0, \tau_{X}\right]$ and it further consists of four scenarios. In scenario (i)(a), there are no claims at all. Therefore, the process $\left\{X_{t}\right\}_{t \geq 0}$ decreases linearly over a length of time $x / c$, and at time $\tau_{X}$ the process $\left\{Y_{t}^{1}\right\}_{t \geq 0}$ is at level $a\left(y_{2}+p_{2} x / c\right) /(1-a)$. This explains (34). Equation (35) in scenario (i)(b) arises when within $\left(0, \tau_{X}\right]$ there are no claims from $\left\{S_{t}\right\}_{t \geq 0}$ (and, hence, we still have $\tau_{X}=x / c$ ), but there is at least one claim from $\left\{L_{t}\right\}_{t \geq 0}$. Therefore, we should make use of the density of $\left\{Y_{t}^{2}\right\}_{t \geq 0}$ at $u$ avoiding ruin given by Proposition 2(ii) at $t=x / c$. For (36) in scenario (i)(c), there is at least one claim from $\left\{S_{t}\right\}_{t \geq 0}$ and, hence, we require the density in Proposition 1(i)(b); this has to be combined with the point mass in Proposition 2(i) since there are no claims from $\left\{L_{t}\right\}_{t \geq 0}$. Equation (37) also follows from a similar argument, simply using the densities in Proposition 1(i)(b) and Proposition 2(ii).

For ruin of $\left\{X_{t}\right\}_{t \geq 0}$ due to jumps, we have (14) (or $Y_{\tau_{X}}^{1}=a Y_{\tau_{X}}^{2} /(1-a)-\left|X_{\tau_{X}}\right| /(1-a)$ ). Note that this case involves at least one claim from $\left\{S_{t}\right\}_{t \geq 0}$, and, therefore, it is analyzed based on the number of claims arising from $\left\{L_{t}\right\}_{t \geq 0}$ only in the interval $\left(0, \tau_{X}\right]$ and it further consists of two scenarios. The detailed explanations of (38) and (39) are omitted here, but one just has to apply (ii) instead of (i) of Proposition 1.

Remark 4. In principle $\mathrm{E}_{\left(y_{1}, y_{2}\right)}\left[\mathrm{e}^{-\beta \tau} \mathbf{1}_{\{\tau<\infty\}}\right]$ can be obtained explicitly via straightforward but tedious integration for mixed Erlang claims.

Remark 5. An alternative expression for $\mathrm{P}_{x}\left(\tau_{X}>t\right)$ can also be obtained from a direct application of Proposition 1, and is given as, for $0<t<x / c$,

$$
\begin{aligned}
\mathrm{P}_{x}\left(\tau_{X}>t\right)= & \int_{t}^{x / c} h_{C}(v \mid x) \mathrm{d} v+\int_{t}^{x / c} \int_{0}^{\infty} h_{J}(z, v \mid x) \mathrm{d} z \mathrm{~d} v \\
& +\mathrm{P}_{x}\left(\tau_{X}=\frac{x}{c},\left|X_{\tau_{X}}\right|=0\right) .
\end{aligned}
$$

Nonetheless, it is obvious that representation (33) is simpler and more tractable than (40). 


\section{The effect of potential ruin of the reinsurer over the cedent}

In the previous section, the distribution of the first ruin time of the two insurers was obtained via its Laplace transform. According to the decomposition in (31) of Proposition 3, we are able to keep track of which insurer ruins first. However, in the case of ruin, it is natural to question what would be the consequence of the ruin of one insurer on the other. Once the bivariate process $\left\{\left(Y_{t}^{1}, Y_{t}^{2}\right)\right\}_{t \geq 0}$ enters $\mathcal{A}^{-}$, then ruin of the process $\left\{Y_{t}^{1}\right\}_{t \geq 0}$ happens no later than the ruin event of the second insurer $\left\{Y_{t}^{2}\right\}_{t \geq 0}$. It would be interesting to know with what probability the ruin of $\left\{Y_{t}^{1}\right\}_{t \geq 0}$ would also lead to the ruin of $\left\{Y_{t}^{2}\right\}_{t \geq 0}$ (through the claims process $\left\{L_{t}\right\}_{t \geq 0}$ ) and the amount of deficit in this case. Unfortunately, this is still an open problem for the moment which is difficult to solve due to the dependence between $\left\{Y_{t}^{1}\right\}_{t \geq 0}$ and $\left\{Y_{t}^{2}\right\}_{t \geq 0}$ (for further comments, see the end of the section). On the other hand, it is possible to obtain information about whether $\left\{Y_{t}^{2}\right\}_{t \geq 0}$ will get ruined before $\left\{Y_{t}^{1}\right\}_{t \geq 0}$ and the surplus level of $\left\{Y_{t}^{1}\right\}_{t \geq 0}$ when such an event occurs. This is particularly important when $\left\{\left(Y_{t}^{1}, Y_{t}^{2}\right)\right\}_{t \geq 0}$ is interpreted as an insurer-reinsurer application, because the reinsurance company needs to be reliable vis-à-vis its clients and needs to give some guarantee to its customers, whereas the insurer or the cedent would like to know its actual surplus if the reinsurer is ruined first, so that appropriate action can be taken.

Geometrically, it is easy to see that ruin of $\left\{Y_{t}^{2}\right\}_{t \geq 0}$ before $\left\{Y_{t}^{1}\right\}_{t \geq 0}$ is possible if and only if $\left(y_{1}, y_{2}\right)$ belongs to $\mathcal{A}^{+}$, and in this case $\left\{\left(Y_{t}^{1}, Y_{t}^{2}\right)\right\}_{t \geq 0}$ remains in $\mathcal{A}^{+}$when $\left\{Y_{t}^{2}\right\}_{t \geq 0}$ drops below 0 . Assuming that $\left(y_{1}, y_{2}\right) \in \mathcal{A}^{+}$, our goal here is to find the distribution of the surplus level of the insurer when the reinsurer ruins, jointly to the event $\left\{\tau_{X}>\tau_{2}\right\}$ (because in this scenario $\left.\tau=\tau_{2}\right)$. Mathematically, we are interested in the quantity $\mathrm{P}_{\left(y_{1}, y_{2}\right)}\left(Y_{\tau_{2}}^{1}>u, \tau_{X}>\tau_{2}\right)$, where $\mathrm{P}_{\left(y_{1}, y_{2}\right)}$ is the conditional probability given the initial surplus levels $\left(Y_{0}^{1}, Y_{0}^{2}\right)=\left(y_{1}, y_{2}\right)$. Note that it is possible for $Y_{\tau_{2}}^{1}$ to be negative because a large claim from $\left\{L_{t}\right\}_{t \geq 0}$ could possibly ruin both $\left\{Y_{t}^{1}\right\}_{t \geq 0}$ and $\left\{Y_{t}^{2}\right\}_{t \geq 0}$ at the same time. Therefore, we will evaluate $\mathrm{P}_{\left(y_{1}, y_{2}\right)}\left(Y_{\tau_{2}}^{1}>\right.$ $u, \tau_{X}>\tau_{2}$ ) for the domain $-\infty<u<\infty$. The result is summarized in the following proposition.

Proposition 4. For the bivariate risk process $\left\{\left(Y_{t}^{1}, Y_{t}^{2}\right)\right\}_{t \geq 0}$, the survival function of the surplus level of $\left\{Y_{t}^{1}\right\}_{t \geq 0}$ when $\left\{Y_{t}^{2}\right\}_{t \geq 0}$ ruins jointly to the event $\left\{\tau_{X}>\tau_{2}\right\}$ is continuous in $u$ and is given by

$$
\begin{aligned}
& \mathrm{P}_{\left(y_{1}, y_{2}\right)}\left(Y_{\tau_{2}}^{1}>u, \tau_{X}>\tau_{2}\right) \\
& \qquad \begin{array}{rll}
\sum_{n=0}^{\infty} \int_{0}^{x / c} \int_{-(1-a) u / a}^{[x-(1-a) u-c t] / a} \mathrm{e}^{-\lambda_{S} t} \frac{\left(\lambda_{S} t\right)^{n}}{n !} \\
\times F_{a, S}^{* n}(x-(1-a) u-c t-a z) \eta\left(z, t \mid y_{2}\right) \mathrm{d} z \mathrm{~d} t & \\
+\sum_{n=0}^{\infty} \int_{0}^{x / c} \int_{0}^{-(1-a) u / a} \mathrm{e}^{-\lambda_{S} t} \frac{\left(\lambda_{S} t\right)^{n}}{n !} F_{a, S}^{* n}(x-c t) & \\
\sum_{n=0}^{\infty} \int_{0}^{[x-(1-a) u] / c} \int_{0}^{[x-(1-a) u-c t] / a} \mathrm{e}^{-\lambda_{S} t} \frac{\left(\lambda_{S} t\right)^{n}}{n !} & \\
\times F_{a, S}^{* n}(x-(1-a) u-c t-a z) \eta\left(z, t \mid y_{2}\right) \mathrm{d} z \mathrm{~d} t, & 0 \leq u<x /(1-a), \\
0, & u \geq x /(1-a) .
\end{array}
\end{aligned}
$$


Here $x=(1-a) y_{1}-a y_{2}>0$ and $\eta\left(z, t \mid y_{2}\right)$ is the density of $\left(\left|Y_{\tau_{2}}^{2}\right|, \tau_{2}\right)$ at $(z, t)$ given $Y_{0}^{2}=y_{2}$, which is available from Corollary 3 of Landriault and Willmot (2009) (see also Dickson (2008)).

Proof. Because

$$
Y_{t}^{1}=\frac{a}{1-a} Y_{t}^{2}+\frac{1}{1-a} X_{t} \quad \text { and } \quad\left\{\tau_{X}>\tau_{2}\right\}=\left\{X_{\tau_{2}}>0\right\}
$$

(recall that $\mathcal{A}^{-}$is absorbing), and since $\left\{X_{t}\right\}_{t \geq 0}$ is independent of $\left\{Y_{t}^{2}\right\}_{t \geq 0}$ and, hence, $\left(\left|Y_{\tau_{2}}^{2}\right|, \tau_{2}\right)$, we obtain

$$
\begin{aligned}
\mathrm{P}_{\left(y_{1}, y_{2}\right)} & \left(Y_{\tau_{2}}^{1}>u, \tau_{X}>\tau_{2}\right) \\
& =\mathrm{P}_{\left(y_{1}, y_{2}\right)}\left(\frac{a}{1-a} Y_{\tau_{2}}^{2}+\frac{1}{1-a} X_{\tau_{2}}>u, X_{\tau_{2}}>0\right) \\
& =\int_{0}^{\infty} \int_{0}^{\infty} \mathrm{P}_{x}\left(-\frac{a}{1-a} z+\frac{1}{1-a} X_{t}>u, X_{t}>0\right) \eta\left(z, t \mid y_{2}\right) \mathrm{d} z \mathrm{~d} t .
\end{aligned}
$$

Using the representation $X_{t}=x-c t-S_{t}^{a}$, the above equation can be rewritten as

$$
\begin{aligned}
\mathrm{P}_{\left(y_{1}, y_{2}\right)} & \left(Y_{\tau_{2}}^{1}>u, \tau_{X}>\tau_{2}\right) \\
= & \int_{0}^{\infty} \int_{0}^{\infty} \mathrm{P}\left(S_{t}^{a}<x-(1-a) u-c t-a z, S_{t}^{a}<x-c t\right) \eta\left(z, t \mid y_{2}\right) \mathrm{d} z \mathrm{~d} t \\
& =\int_{0}^{\infty} \int_{0}^{\infty} \mathrm{P}\left(S_{t}^{a}<\min (x-(1-a) u-c t-a z, x-c t)\right) \eta\left(z, t \mid y_{2}\right) \mathrm{d} z \mathrm{~d} t .
\end{aligned}
$$

Note that the probability term in the above integrand is 0 for some combinations of $z$ and $t$. Moreover, we need to distinguish between the cases $u \geq 0$ and $u<0$.

Case 1: $u \geq 0$. We have $\min (x-(1-a) u-c t-a z, x-c t)=x-(1-a) u-c t-a z$ since $z$ is positive, and, therefore,

$$
\begin{aligned}
\mathrm{P}\left(S_{t}^{a}<\right. & \min (x-(1-a) u-c t-a z, x-c t)) \\
= & \mathrm{P}\left(S_{t}^{a}<x-(1-a) u-c t-a z\right) \\
= & \sum_{n=0}^{\infty} \mathrm{e}^{-\lambda_{S} t} \frac{\left(\lambda_{S} t\right)^{n}}{n !} F_{a, S}^{* n}(x-(1-a) u-c t-a z) \\
& -\mathrm{e}^{-\lambda_{S} t} \mathbf{1}_{\{x-(1-a) u-c t-a z=0\} .}
\end{aligned}
$$

We remark that the last correction term at the end is to adjust for the fact that $\mathrm{P}\left(S_{t}^{a}<v\right)=$ $\mathrm{P}\left(S_{t}^{a} \leq v\right)$ for all $-\infty<v<\infty$ except for $v=0$ due to the point mass arising from $S_{t}^{a}=0$. Clearly, the above expression is 0 if $x-(1-a) u-c t-a z \leq 0$. Since both $z$ and $t$ are positive, $x-(1-a) u-c t-a z$ is less than 0 if $x-(1-a) u \leq 0$. This explains the final equation in (41) for $u \geq x /(1-a)$. The integration domain in (42) for which (43) is generally nonzero is given by $\{(z, t): c t+a z<x-(1-a) u\}$ when $x-(1-a) u>0$. By noting that $\{(z, t): c t+a z=x-(1-a) u\}$ is a set of measure 0 , we can ignore the correction term in (43). Combining these observations leads to the second equation in (41) for $0 \leq u<x /(1-a)$.

Case 2: $u<0$. Note that $x-(1-a) u$ is always positive. Moreover, we have

$$
\min (x-(1-a) u-c t-a z, x-c t)= \begin{cases}x-(1-a) u-c t-a z, & z \geq-(1-a) u / a, \\ x-c t, & z<-(1-a) u / a .\end{cases}
$$


Hence, for $z \geq-(1-a) u / a$, representation (43) still holds true and the relevant domain of integration in (42) is $\{(z, t): c t+a z<x-(1-a) u, z \geq-(1-a) u / a\}$. On the other hand, for $z<-(1-a) u / a$,

$$
\begin{aligned}
\mathrm{P}\left(S_{t}^{a}\right. & <\min (x-(1-a) u-c t-a z, x-c t)) \\
& =\mathrm{P}\left(S_{t}^{a}<x-c t\right) \\
& =\sum_{n=0}^{\infty} \mathrm{e}^{-\lambda_{S} t} \frac{\left(\lambda_{S} t\right)^{n}}{n !} F_{a, S}^{* n}(x-c t)-\mathrm{e}^{-\lambda_{S} t} \mathbf{1}_{\{x-c t=0\}},
\end{aligned}
$$

which is generally nonzero for $\{(z, t): x-c t>0, z<-(1-a) u / a\}$. Taking into account the above two contributions in the integrand of (42) (and again ignoring the terms on sets with measure 0 ), we arrive at the first equation in (41) for $u<0$.

Note that Proposition 4 is concerned with the surplus level of $\left\{Y_{t}^{1}\right\}_{t \geq 0}$ when $\left\{Y_{t}^{2}\right\}_{t \geq 0}$ is ruined within the set $\mathcal{A}^{+}$(i.e. $\tau=\tau_{2}$ ). An open problem would be

- how to find the distribution of the surplus level of $\left\{Y_{t}^{2}\right\}_{t \geq 0}$ when $\left\{Y_{t}^{1}\right\}_{t \geq 0}$ is ruined (for the first time) within the set $\mathcal{A}^{-}$(i.e. $\tau=\tau_{1}$ ).

We remark that regardless of whether ruin of the bivariate process $\left\{\left(Y_{t}^{1}, Y_{t}^{2}\right)\right\}_{t \geq 0}$ occurs from $\mathcal{A}^{+}$or $\mathcal{A}^{-}$, it is possible that $\tau=\tau_{1}=\tau_{2}$ due a large claim from $\left\{L_{t}\right\}_{t \geq 0}$. If ruin of $\left\{Y_{t}^{2}\right\}_{t \geq 0}$ occurs within $\mathcal{A}^{+}$, the probability of the event $\left\{\tau=\tau_{1}=\tau_{2}\right\}$ is given by

$$
\mathrm{P}_{\left(y_{1}, y_{2}\right)}\left(Y_{\tau_{2}}^{1}<0, \tau_{X}>\tau_{2}\right)=\mathrm{P}_{\left(y_{1}, y_{2}\right)}\left(\tau_{X}>\tau_{2}\right)-\mathrm{P}_{\left(y_{1}, y_{2}\right)}\left(Y_{\tau_{2}}^{1}>0, \tau_{X}>\tau_{2}\right),
$$

where $\mathrm{P}_{\left(y_{1}, y_{2}\right)}\left(\tau_{X}>\tau_{2}\right)$ can be retrieved from (32) at $\beta=0$ and $\mathrm{P}_{\left(y_{1}, y_{2}\right)}\left(Y_{\tau_{2}}^{1}>0, \tau_{X}>\tau_{2}\right)$ from (41) at $u=0$. In a similar manner, if the aforementioned open problem is solved, one could also obtain the probability of the event $\left\{\tau=\tau_{1}=\tau_{2}\right\}$ for ruin of $\left\{Y_{t}^{1}\right\}_{t \geq 0}$ within the set $\mathcal{A}^{-}$.

Since the event $\left\{\tau=\tau_{1}=\tau_{2}\right\}$ is caused by a large claim from $\left\{L_{t}\right\}_{t \geq 0}$, a related problem would be

- how to determine the distribution of the claim causing ruin in such a case.

This is of particular importance because such a claim has an adverse effect on both insurers at the same time and the associated risk has to be assessed properly. On the other hand, one might also be interested in the problem of

- how to find the joint distribution of $\left(Y_{\tau}^{1}, Y_{\tau}^{2}\right)$, which represents the surplus levels of the two lines at the time of ruin $\tau$.

We remark that a similar problem has been considered in the form of a Gerber-Shiu function (see Gerber and Shiu (1998)) in Gong et al. (2010) in a different two-dimensional risk model using recursive methods. We leave these as open problems in the current model.

\section{Acknowledgements}

Andrei Badescu gratefully acknowledges financial support from the Natural Sciences and Engineering Research Council of Canada (NSERC). Eric Cheung also acknowledges a start-up fund provided by the Faculty of Science and the Department of Statistics and Actuarial Science, as well as the Seed Funding for Basic Research (project number 201103159001) provided by the University Research Committee at the University of Hong Kong. 


\section{References}

Avram, F., Palmowski, Z. and Pistorius, M. (2008a). A two-dimensional ruin problem on the positive quadrant. Insurance Math. Econom. 42, 227-234.

Avram, F., Palmowski, Z. And Pistorius, M. R. (2008b). Exit problem of a two-dimensional risk process from the quadrant: exact and asymptotic results. Ann. Appl. Prob. 18, 2421-2449.

Bertoin, J. (1997). Exponential decay and ergodicity of completely asymmetric Lévy processes in a finite interval. Ann. Appl. Prob. 7, 156-169.

CAI, J. AND LI, H. (2005). Multivariate risk model of phase type. Insurance Math. Econom. 36, 137-152.

CAI, J. AND LI, H. (2007). Dependence properties and bounds for ruin probabilities in multivariate compound risk models. J. Multivariate Anal. 98, 757-773.

Chan, W.-S., YANG, H. AND Zhang, L. (2003). Some results on ruin probabilities in a two-dimensional risk model. Insurance Math. Econom. 32, 345-358.

Cheung, E. C. K. And Landriault, D. (2010). A generalized penalty function with the maximum surplus prior to ruin in a MAP risk model. Insurance Math. Econom. 46, 127-134.

DANG, L., ZHU, N. AND Zhang, H. (2009). Survival probability for a two-dimensional risk model. Insurance Math. Econom. 44, 491-496.

DiCKSON, D. C. M. (2008). Some explicit solutions for the joint density of the time of ruin and the deficit at ruin. ASTIN Bull. 38, 259-276.

Dickson, D. C. M. And Willmot, G. E. (2005). The density of the time to ruin in the classical Poisson risk model. ASTIN Bull. 35, 45-60.

Gerber, H. U. And Shiu, E. S. W. (1998). On the time value of ruin. N. Amer. Actuarial J. 2, 48-78.

Gong, L., Badescu, A. L. And Cheung, E. C. K. (2010). Recursive methods for a two-dimensional risk process with common shocks. Submitted.

Landriault, D. and Willmot, G. E. (2009). On the joint distributions of the time to ruin, the surplus prior to ruin, and the deficit at ruin in the classical risk model. N. Amer. Actuarial J. 13, 252-279.

LI, J., LIU, Z. AND TANG, Q. (2007). On the ruin probabilities of a bidimensional perturbed risk model. Insurance Math. Econom. 41, 185-195.

Lin, X. S. AND Willmot, G. E. (1999). Analysis of a defective renewal equation arising in ruin theory. Insurance Math. Econom. 25, 63-84.

Rabehasaina, L. (2009). Risk processes with interest force in Markovian environment. Stoch. Models 25, 580-613.

Suprun, V. N. (1976). Problem of destruction and resolvent of a terminating process with independent increments. Ukrainian Math. J. 28, 39-45.

Tıuss, H. C. (1994). Stochastic Models. John Wiley, Chichester.

Willmot, G. E. And Woo, J.-K. (2007). On the class of Erlang mixtures with risk theoretic applications. N. Amer. Actuarial J. 11, 99-115.

Yuen, K. C., GuO, J. AND Wu, X. (2006). On the first time of ruin in the bivariate compound Poisson model. Insurance Math. Econom. 38, 298-308. 